\section{Increased Rrm2 gene dosage reduces fragile site breakage and prolongs survival of ATR mutant mice}

\author{
Andres J. Lopez-Contreras, ${ }^{1,7,8}$ Julia Specks, 1,8 \\ Jacqueline H. Barlow, ${ }^{2}$ Chiara Ambrogio, ${ }^{3}$ \\ Claus Desler, ${ }^{4}$ Svante Vikingsson, ${ }^{5}$ \\ Sara Rodrigo-Perez, ${ }^{1}$ Henrik Green, ${ }^{5,6}$ \\ Lene Juel Rasmussen, ${ }^{4}$ Matilde Murga, ${ }^{1}$ \\ André Nussenzweig, ${ }^{2}$ \\ and Oscar Fernandez-Capetillo ${ }^{1}$
}

\begin{abstract}
${ }^{1}$ Genomic Instability Group, Spanish National Cancer Research Centre (CNIO), Madrid 28029, Spain; ${ }^{2}$ Laboratory of Genome Integrity, National Cancer Institute, National Institutes of Health, Bethesda, Maryland 20892, USA; ${ }^{3}$ Experimental Oncology Group, Spanish National Cancer Research Centre (CNIO), Madrid 28029, Spain; ${ }^{4}$ Center for Healthy Aging, Department of Cellular and Molecular Medicine, University of Copenhagen, 2200 Copenhagen N, Denmark; ${ }^{5}$ Division of Drug Research/Clinical Pharmacology, Department of Medical and Health Sciences, Linköping University, SE-581 85 Linköping, Sweden; ${ }^{6}$ Department of Forensic Genetics and Forensic Toxicology, National Board of Forensic Medicine, SE-581 85 Linköping, Sweden
\end{abstract}

In Saccharomyces cerevisiae, absence of the checkpoint kinase Mec1 (ATR) is viable upon mutations that increase the activity of the ribonucleotide reductase (RNR) complex. Whether this pathway is conserved in mammals remains unknown. Here we show that cells from mice carrying extra alleles of the RNR regulatory subunit RRM2 (Rrm $\left.2^{\text {TG }}\right)$ present supraphysiological RNR activity and reduced chromosomal breakage at fragile sites. Moreover, increased Rrm2 gene dosage significantly extends the life span of ATR mutant mice. Our study reveals the first genetic condition in mammals that reduces fragile site expression and alleviates the severity of a progeroid disease by increasing RNR activity.

Supplemental material is available for this article.

Received December 5, 2014; revised version accepted March 2, 2015.

Replication stress (RS) has emerged as a source of genome instability in human diseases, including cancer and premature aging (Lecona and Fernandez-Capetillo 2014; Zeman and Cimprich 2014). In brief, RS is defined by the accumulation of abnormal amounts of ssDNA at

[Keywords: ATR; fragile site; mouse models; RNR; replication stress] Present address: Center for Chromosome Stability, Department of Cellular and Molecular Medicine, Panum Institute, University of Copenhagen, 2200 Copenhagen N, Denmark.

${ }^{8}$ These authors contributed equally to this work.

Corresponding authors: ofernandez@cnio.es, ajlopez@sund.ku.dk

Article is online at http://www.genesdev.org/cgi/doi/10.1101/gad.256958. 114. stalled replication forks that, due to its recombinogenic nature, can initiate genomic rearrangements. In mammals, RS is sensed and suppressed by a signaling cascade initiated by the ATR kinase, which, together with its target kinase, CHK1, suppresses RS through the phosphorylation of multiple targets (Cimprich and Cortez 2008; Lopez-Contreras and Fernandez-Capetillo 2010). ATR and $\mathrm{CHK} 1$ are essential for embryonic development in mice (Brown and Baltimore 2000; de Klein et al. 2000; Liu et al. 2000), which is due to the role of the RS response (RSR) in preventing replication-born chromosome breakage. Whether the RSR protects all forks or a subset of them during replication is unclear. On the one hand, proteomic studies of the human replisome in unchallenged conditions have failed to detect ATR or CHK1 in the vicinity of replication forks (Lopez-Contreras et al. 2013; Sirbu et al. 2013), suggesting that their activity might be particularly necessary for only a subset of forks, such as damaged ones. Accordingly, chromosomal breaks that arise upon ATR inactivation locate preferentially at specific loci named common fragile sites (CFSs) (Casper et al. 2002) and early replicating fragile sites (ERFSs) (Barlow et al. 2013). Regardless of whether ATR works at all forks or only some of them, how it suppresses RS and why it is essential are still not fully understood.

Ribonucleotide reductase (RNR) is a tetrameric enzyme composed of two catalytic (RRM1, Rnr1 in yeast) and two regulatory (RRM2, Rnr2 in yeast) subunits (Jordan and Reichard 1998). It reduces NDPs into dNDPs, which is a rate-limiting step for the production of dNTPs. In yeast, the lethality of mec1 $1 \Delta$ strains can be bypassed by mutations that increase RNR activity. The first evidence of a connection between ATR and RNR came from the discovery of Crt1 (a transcriptional repressor of RNR subunits) as a suppressor of Mec1 lethality in Saccharomyces cerevisiae (Huang et al. 1998). Furthermore, overproduction of Rnrl was shown to be sufficient to rescue mec1s viability (Desany et al. 1998; Vallen and Cross 1999). In addition to the transcriptional regulation provided by Crt1, Mec1 and its downstream kinase, Dun1, also increase RNR activity by phosphorylating the RNR inhibitor Sml1, leading to its degradation (Zhao et al. 2001; Zhao and Rothstein 2002). As in the case of Crt1, sml1 deletion also rescued mec1s viability (Zhao et al. 1998). Finally, the activity of yeast RNR is also regulated through the control of Rnr2 localization. S. cerevisiae Dif1 or Schizosaccharomyces pombe Spd1 retain Rnr2 in the nucleus, preventing its interaction with cytoplasmic Rnr1. Like Crt1 or Sml1, deletion of spd1 or dif1 increases RNR activity and suppresses the lethality of checkpoint mutants (Liu et al. 2003; Lee et al. 2008; Wu and Huang 2008). Even though no clear orthologs for yeast RNR inhibitory proteins have been found in mammals, evidence suggests that the connection between ATR and RNR is conserved to some extent.

First, the addition of nucleosides has been shown to reduce RS in various instances, such as in response to

(c) 2015 Lopez-Contreras et al. This article is distributed exclusively by Cold Spring Harbor Laboratory Press for the first six months after the full-issue publication date (see http://genesdev.cshlp.org/site/misc/ terms.xhtml). After six months, it is available under a Creative Commons License (Attribution-NonCommercial 4.0 International), as described at http://creativecommons.org/licenses/by-nc/4.0/. 

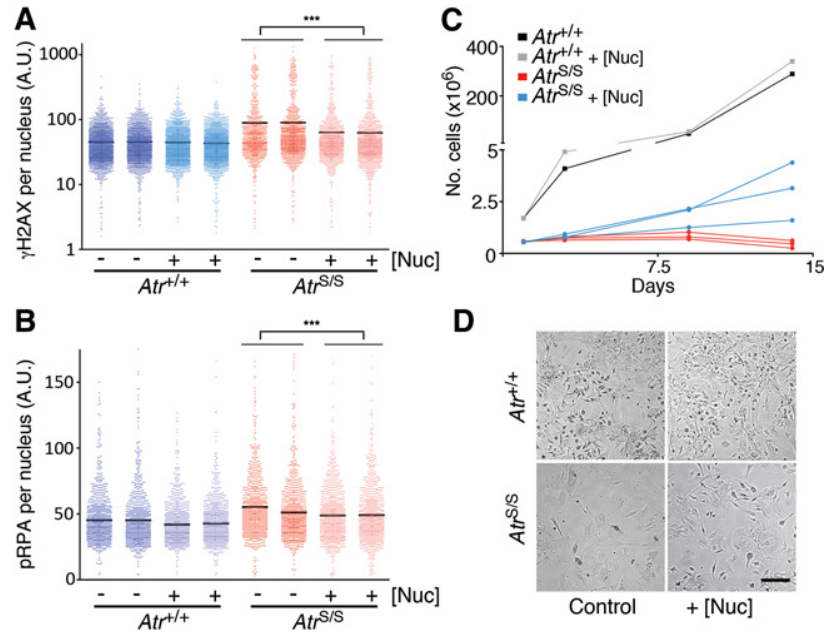

D

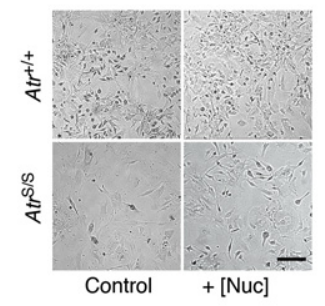

Figure 1. Nucleoside supplementation reduces RS and improves growth of ATR-Seckel MEFs. (A) HTM-mediated quantification of the intensity of $\gamma \mathrm{H} 2 \mathrm{AX}$ per individual nucleus on $\mathrm{Atr}^{+/+}$and $\mathrm{Atr}^{\mathrm{S} / \mathrm{S}}$ MEFs treated or not with $60 \mu \mathrm{M}$ nucleosides for $24 \mathrm{~h}$. Data are representative of two independent cell lines. $(B)$ HTM-mediated quantification of the intensity of phosphorylated RPA per individual nucleus on $\mathrm{Atr}^{+/+}$and $\mathrm{Atr}{ }^{S / S}$ MEFs treated or not with $60 \mu \mathrm{M}$ nucleosides for $24 \mathrm{~h}$. Data are representative of two independent cell lines. (C) Proliferation curves of $\mathrm{Atr}^{+/+}$and $A t r^{S / S}$ MEFs grown in the presence or absence of $60 \mu \mathrm{M}$ nucleoside supplementation. Data are representative of two independent analyses. $(D)$ Representative pictures of wild-type and ATR-Seckel MEFs at the end point of the proliferation assay shown in $C$. Note that while all ATR mutant MEFs show the flattened morphology of senescent cells at this point, those treated with a nucleoside supplement still present cells with the elongated morphology of proliferating fibroblasts. $\left({ }^{* *}\right) P<0.001$.

oncogenes or during zebrafish development (Bester et al. 2011; Danilova et al. 2014). Furthermore, CHK1 activation by topoisomerase inhibitors induces the expression of RRM2 through E2F-dependent transcription (Zhang et al. 2009). In addition, p53 induces the expression of an alternative regulatory subunit (RRM2B or p53R2). However, both of these activities occur only after a prolonged exposure to DNA damage and cannot account for the more immediate role that ATR plays during DNA replication. Regarding Sml1, a recent study reported that IRBIT, a protein known to be involved in ion transport, shares some distant homology with Sml1 in a small fragment of its sequence (Arnaoutov and Dasso 2014). This fragment can bind and inhibit the RNR complex, but its activity seems to be restricted to mitosis and RRM1/RRM2B complexes. In summary, while these experiments suggest that RNR regulation might suppress RS in mammals, whether and how these functions are linked to ATR activity are not understood.

Here we sought to explore the impact of the RNR in the ATR response in mammals. To this end, we generated a mouse model with increased levels of the regulatory subunit RRM2 (Rrm2 $\left.{ }^{\mathrm{TG}}\right)$. We show that Rrm2 transgenic mice present supraphysiological RNR activity, which becomes protective in the context of insufficient dNTP levels. Importantly, we show that increased levels of RRM2 can limit chromosome breakage in response to ATR inhibitors and extend the life span of mice with reduced ATR levels. Altogether, our findings provide strong genetic support for the concept that regulating nucleotide pools is one of the key functions of ATR in mammals.

\section{Results and Discussion}

Nucleosides limit RS and improve growth of ATR-Seckel mouse embryonic fibroblasts (MEFs)

To explore whether increased nucleotides could suppress phenotypes related to ATR deficiency in mammals, we used MEFs from a model of the ATR-Seckel syndrome (Murga et al. 2009). ATR-Seckel MEFs present severely reduced ATR levels and undergo premature senescence due to the accumulation of RS (Murga et al. 2009; Monasor et al. 2013). While nucleotides are not cell-permeable, a recent report showed that the addition of nucleosides, the nucleotide precursors, to the culture medium alleviates oncogene-induced RS (Bester et al. 2011). In the case of ATR-Seckel MEFs, the addition of nucleosides significantly reduced the levels of RS, as quantified by measuring the phosphorylation status of histone H2AX $(\gamma \mathrm{H} 2 \mathrm{AX})$ or the ssDNA-binding protein RPA by highthroughput microscopy (HTM) (Fig. 1A,B). In addition, nucleoside supplementation to the culture medium partially rescued the growth defect in three independent lines of ATR-Seckel MEFs (Fig. 1C,D). These results suggest that increased levels of nucleotides can suppress the RS that derives from reduced ATR activity.

\section{Generation of a mouse model with increased RRM2 levels}

To investigate the consequences of increased RNR activity in a mammalian organism, we generated mice carrying extra alleles of the regulatory subunit RRM2. We chose to focus on RRM2 because of the following considerations. First, knockdown of RRM2 increased RS in response to the RNR inhibitor hydroxyurea (HU) in human U2OS cells, whereas overexpression had the opposite effect (Fig. 2A,B; Supplemental Fig. S1). Second, available microarray analyses revealed reduced levels of RRM2 expression on ATR-Seckel embryos (Murga et al. 2009), suggesting that a defective RNR activity might contribute to the phenotypes of these mice. Third, ATR-dependent degradation of cyclin $\mathrm{F}$ has been shown to increase RRM2 levels in response to DNA damage (D'Angiolella et al. 2012). Finally, classic reports described HU-resistant hamster cell lines that were associated with genomic amplification of Rrm2, but not Rrm1, which led to increased RNR activity in these cells (Lewis and Wright 1974; Tonin et al. 1987). Hence, we hypothesized that mice carrying additional copies of the Rrm2 gene would similarly have increased RNR activity. To preserve the endogenous regulation of Rrm2 transcription, we subcloned a 26-kb region encompassing the Rrm2 gene and $10 \mathrm{~kb}$ of flanking sequences (Fig. 2C). This strategy, in contrast to classic transgenesis using plasmids harboring cDNA, has been proven successful for generating animals with increased activity of genes that are otherwise deleterious when aberrantly overexpressed, such as Chk1 or Tp53 (Garcia-Cao et al. 2002; Lopez-Contreras et al. 2012).

Transgenic lines were identified by Southern blotting, and the integration site was subsequently mapped by inverse PCR. We selected one line $\left(\right.$ Rrm $\left.2^{\mathrm{TG}}\right)$ carrying several alleles integrated in tandem (Fig. 2D) on the basis of having the insertion of the transgene at a locus where it did not disrupt any other gene (chr12: 118,863,235). Mapping the insertion also allowed for genotyping of homozygous or heterozygous mice (Fig. 2E). Rrm2 transgenic 

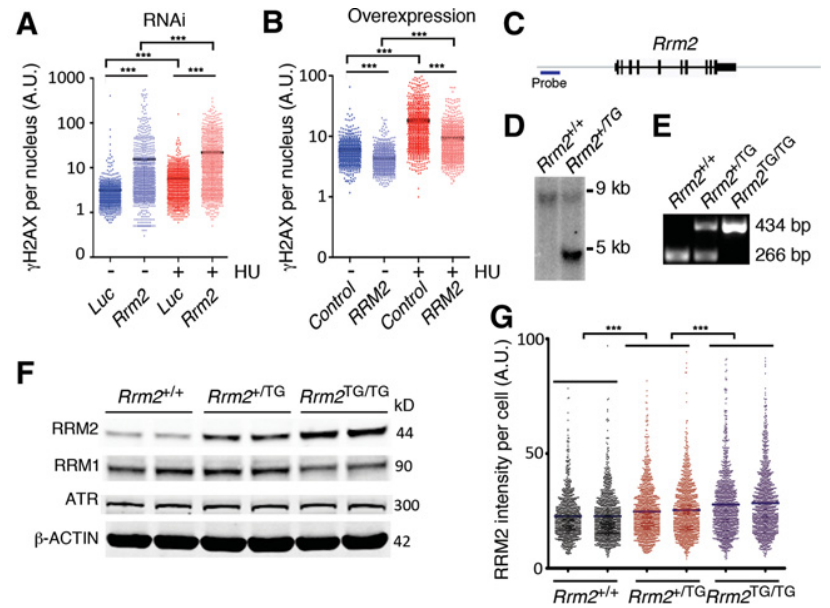

Figure 2. Generation of $R r m 2^{\mathrm{TG}}$ mice. $(A)$ HTM-mediated quantification of the intensity of $\gamma \mathrm{H} 2 \mathrm{AX}$ per individual nucleus on human U2OS cells $48 \mathrm{~h}$ after being transfected with endogenous siRNAs (esiRNAs) targeting either firefly luciferase (Luc) or Rrm2. Where indicated, cells were also exposed to $2 \mathrm{mM}$ HU for $3 \mathrm{~h}$. Data are representative of two independent experiments. (B) HTM-mediated quantification of the intensity of $\gamma \mathrm{H} 2 \mathrm{AX}$ per individual nucleus on human U2OS cells $48 \mathrm{~h}$ after being transfected with a RRM2-expressing plasmid or the empty vector (control). Where indicated, cells were also exposed to $2 \mathrm{mM} \mathrm{HU}$ for $3 \mathrm{~h}$. Data are representative of two independent experiments. (C) Scheme of the genomic locus (including the Rrm2 gene and flanking regions) that was used for the generation of Rrm $2^{\mathrm{TG}}$ mice. $(D)$ Southern blot illustrating the presence of a single transgene integration site $(5 \mathrm{~kb})$ on the Rrm $2^{\mathrm{TG}}$ strain. The $9-\mathrm{kb}$ band corresponds to the endogenous Rrm2 gene. $(E)$ Genotyping PCR illustrating the three genotypes that can be obtained at Mendelian ratios on the $R r m 2^{\mathrm{TG}}$ strain. $(F)$ Western blot illustrating the levels of RRM2, RRM1, and ATR on Rrm2 ${ }^{+/+}, \mathrm{Rrm}^{+/ \mathrm{TG}}$, and $\mathrm{Rrm}^{\mathrm{TG} / \mathrm{TG}}$ MEFs. $\beta$-Actin was used as a loading control. $(G)$ HTM-mediated quantification of the intensity of RRM2 per individual cell on $\mathrm{Rrm}^{+/+}, \mathrm{Rrm}_{2}{ }^{+/ \mathrm{TG}}$, and $\mathrm{Rrm} 2^{\mathrm{TG} / \mathrm{TG}}$ MEFs. RRM2 Western blot of tissues from wild-type and $R r m 2^{\mathrm{Tg}}$ mice. $\left(^{* * *}\right) P<0.001$.

MEFs exhibited increased RRM2 protein levels in a transgene dosage-dependent manner, as detected by Western blotting and immunofluorescence (Fig. 2F,G). In contrast, no effect on the levels of RRM1 or ATR was observed. In tissues, RRM2 expression was analyzed by immunohistochemistry (IHC). RRM2 expression was highest in tissues with active turnover, such as spleen or testes, and increased on Rrm2 transgenic tissues (Supplemental Fig. S2). Interestingly, RRM2 was cytoplasmic in all tissues except testes, where it showed a nuclear distribution consistent with nuclear RNR activity being particularly relevant for DNA repair (D'Angiolella et al. 2012). Regardless of its increased expression, the intracellular localization of RRM2 or its relative abundance between different tissues was not altered on $\mathrm{Rrm} 2^{\mathrm{TG}}$ mice. Thus, mice carrying extra alleles of Rrm2 provide a physiological model to investigate the consequences of increased RRM2 levels.

\section{Increased RNR activity in $\operatorname{Rrm} 2^{T G}$ MEFs}

Two independent methods for quantifying dNTP levels failed to detect significant changes on Rrm2 transgenic MEFs (Supplemental Fig. S3). The absence of an increase in steady-state dNTP levels is in agreement with the existence of feedback inhibitory loops, which inhibit RNR activity when dATP levels increase above physio- logical levels (Jordan and Reichard 1998). Nevertheless, our finding is in agreement with previous studies. For instance, HU-resistant mouse mammary tumor cells with a 40-fold overexpression of RRM2 have normal dNTP levels (Eriksson et al. 1984). In addition, mouse 3T6 cells made HU-resistant upon a gradual increase in HU concentrations have normal levels of dNTPs despite having a threefold to 15-fold increase in RNR activity (Akerblom et al. 1981). Hence, while steady-state dNTP levels are kept at normal levels on Rrm2 transgenic cells, they could harbor increased RNR activity that would be particularly relevant in conditions of limited nucleotide availability.

To examine whether Rrm2 transgenic cells had increased RNR activity, we tested how they responded to the RNR inhibitor HU. A number of independent experiments validated that Rrm2 transgenic cells had increased RNR activity. First, HTM analyses revealed that $R r m 2^{\text {TG }}$ MEFs exhibited lower levels of $\gamma \mathrm{H} 2 \mathrm{AX}$ in response to $\mathrm{HU}$ (Fig. 3A). While Rrm2 $2^{\mathrm{TG} / \mathrm{TG}}$ MEFs express more RRM2 than heterozygous cells, this did not translate into a higher resistance. This could indicate that the strategy of increasing RRM2 might reach a limit if not done together with a concomitant increase in RRM1. Besides H2AX, Rrm2 transgenic MEFs also presented lower levels of RPA phosphorylation in response to HU (Fig. 3B). In
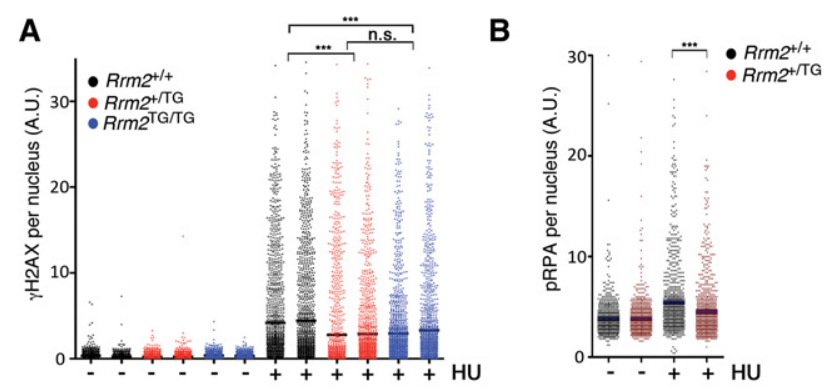

C
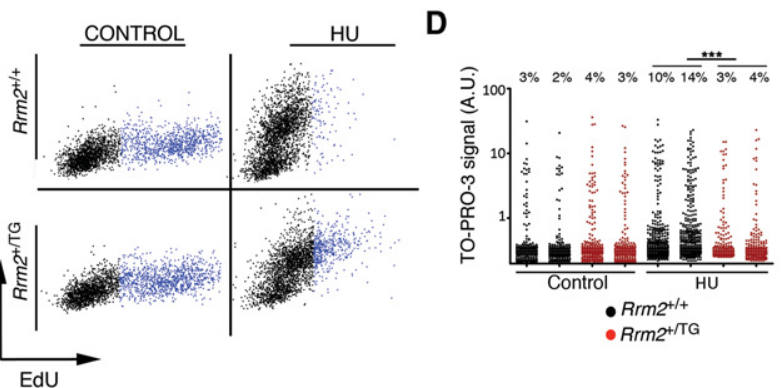

Figure 3. Increased RNR activity on Rrm $2^{\mathrm{TG}}$ cells. $(A)$ HTM-mediated quantification of the intensity of $\gamma \mathrm{H} 2 \mathrm{AX}$ per individual nucleus on $\operatorname{Rrm}^{+/+}, \operatorname{Rrm}^{+/ \mathrm{TG}}$, and Rrm2 ${ }^{\mathrm{TG} / \mathrm{TG}}$ MEFs. Data are representative of three independent experiments. Where indicated, cells were also exposed to $0.5 \mathrm{mM}$ HU for $4 \mathrm{~h}$. (B) HTM-mediated quantification of the intensity of phosphorylated RPA per individual nucleus on $\mathrm{Rrm2}^{+/+}$and $\mathrm{Rrm}^{+/ \mathrm{TG}}$ MEFs. Data are representative of three independent experiments. Where indicated, cells were also exposed to $0.5 \mathrm{mM} \mathrm{HU}$ for $4 \mathrm{~h}$. Data are representative of three independent analyses in four different MEF pairs. (C) HTM-mediated quantification of $\gamma \mathrm{H} 2 \mathrm{AX}$ and EdU intensities per individual nucleus in wild-type and Rrm2 transgenic MEFs treated or not with $0.3 \mathrm{mM}$ HU for $4 \mathrm{~h}$. EdU was added to the cultures for the last $30 \mathrm{~min}$ of the experiment. Blue dots represent EdU-incorporating cells. (D) HTM-mediated quantification of cell death by analysis the incorporation of the TOPRO-3 dye in $\mathrm{Rrm}^{+/+}$and $\mathrm{Rrm}^{+/ \mathrm{TG}}$ treated or not with $0.3 \mathrm{mM}$ $\mathrm{HU}$ for $24 \mathrm{~h}$ (see the Materials and Methods). The experiment was done in triplicate with three independent MEF pairs. $\left(^{* * *}\right) P<0.001$. 
addition to RS markers, Rrm2 transgenic cells were able to incorporate EdU at doses of HU that fully prevented replication in wild-type cells (Fig. 3C). Interestingly, EdU incorporation in Rrm2 ${ }^{+/ T G}$ MEFs could even be detected in cells that presented H2AX phosphorylation. Of note, the actual degree of HU-resistant replication might be higher than observed with this assay, since higher dNTP levels on Rrm2 transgenic cells exposed to HU would compete with EdU during replication. Finally, we tested actual HU resistance by looking at HU-induced cytotoxicity upon prolonged exposure to the drug. Once again, increased levels of RRM2 correlated with lower levels of HU-induced cell death in transgenic cells (Fig. 3D). In contrast, Rrm2 transgenic cells were not protected against other RS-inducing agents, such as aphidicolin or camptothecin, suggesting that increased RRM2 levels become particularly relevant in the context of conditions that limit dNTP levels, such as HU or ATR inhibitors (see below). Of note, neither the Rrm2 transgene nor the supplementation of nucleosides shown above affected cell cycle progression. Altogether, these experiments demonstrate that Rrm $2^{\mathrm{TG}}$ cells present increased RNR activity, which protects them from HU-induced RS and might be beneficial in the context of conditions that reduce dNTP availability.

\section{Rrm $2^{T G}$ protects from reduced ATR activity in vitro and in vivo}

As mentioned, evidence from yeast suggests that stimulating RNR activity is one of the essential activities of Mec1 (ATR). To determine whether a similar interaction might be conserved in mammals, we first tested whether Rrm $2^{\mathrm{TG}}$ cells were resistant to the replicative damage induced by ATR inhibitors previously developed in our group (Toledo et al. 2011). To do this, we looked at the presence of chromosomal abnormalities at CFS and ERFS loci, both of which are sites of preferential chromosomal fragility upon ATR inhibition (Casper et al. 2002; Barlow et al. 2013). Proliferating B lymphocytes from Rrm2 ${ }^{\mathrm{TG}}$ cells presented an overall reduction in the number of chromosome breaks that were induced by ATR inhibitors (Fig. 4A), a difference that was pronounced at CFS and ERFS fragile loci (Fig. 4B,C). Remarkably, ATR inhibitors lead to a reduction in cellular dNTP levels (Supplemental Fig S4), which might contribute to the toxicity of these compounds. Supporting this view, increased levels of RRM2 limit the chromosomal breakage that arises upon ATR inhibition in vitro.

Finally, to determine whether increased RNR activity could also rescue the phenotypes associated with deficient ATR signaling in a mammalian organism, we crossed Rrm2 ${ }^{\mathrm{TG}}$ mice into the ATR-Seckel strain. The Seckel syndrome was originally named "bird-headed dwarfism" because of the overall reduced size and the craniofacial anomalies present in these patients (Seckel 1960). Both of these clinical manifestations, which are recapitulated on ATR-Seckel mice, were significantly alleviated by the Rrm2 transgene (Fig. 4D,E). In addition to the overall appearance, Seckel mice succumb prematurely due to accelerated aging (Murga et al. 2009). Importantly, the presence of the Rrm2 transgene significantly increased the survival of ATR-Seckel mice (Fig. 4F). Atr ${ }^{\mathrm{S} / \mathrm{S}}$; Rrm2 $2^{\mathrm{TG} /+}$ animals doubled the median life span of $\mathrm{Atr} \mathrm{S}^{\mathrm{S} / \mathrm{S}}$; Rrm2 ${ }^{+/+}$mice (50 wk vs. 24 wk). Moreover, the presence of

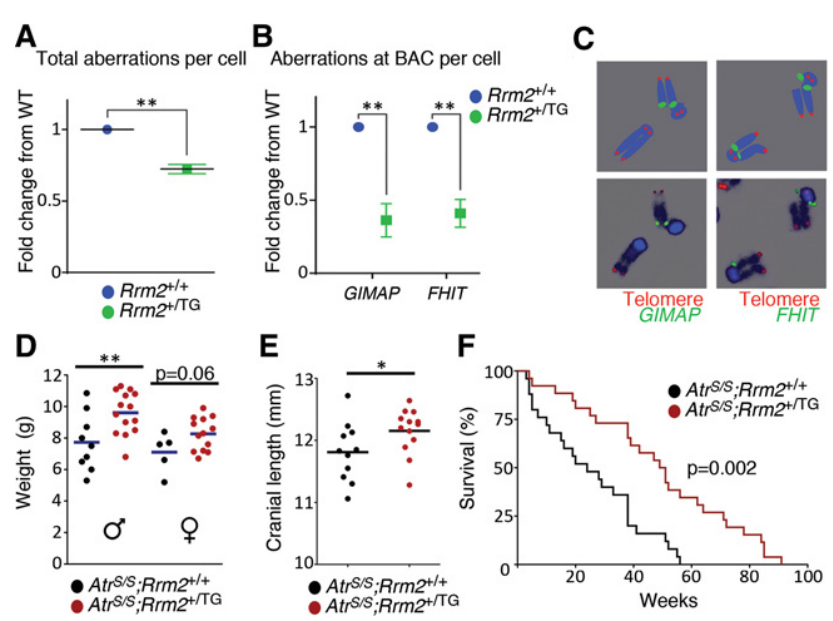

Figure 4. Increased RNR activity limits chromosome breakage in response to ATR inhibition and prolongs survival of ATR-Seckel mice. (A) DNA aberrations per metaphase were counted from $\mathrm{Rrm}^{+/+}$and Rrm2 $^{+/ T G}$ B lymphocytes treated with $10 \mu \mathrm{m}$ ATR inhibitor for 16 h. Data were normalized as fold change from wild-type levels to account for fluctuations between three independent experimental runs. (B) Aberrations at the GIMAP ERFS and the FHIT (FRA14A2) per metaphase normalized as fold change from wild-type levels from the metaphases used in $A$. Aberrations included chromatid and chromosome breaks, radials, and dicentric chromosomes. At least 84 metaphases were scored per replicate. (C, top panel) Diagram of FISH probes, with the DNA in blue, telomeres in red, and BAC probes in green. The bottom panel shows representative rearrangements at GIMAP and FHIT. (D) The weight of $A T R^{\mathrm{S} / \mathrm{s}} ; \mathrm{Rrm}^{+/+}$and $A T R^{\mathrm{S} / \mathrm{S}}$;Rrm2 $2^{+/ \mathrm{TG}}$ male (left) and female (right) mice at $10 \mathrm{wk}$ of age. (E) The cranial length of $A T R^{\mathrm{S} / \mathrm{S}} ; \operatorname{Rrm}^{+/+}$and $A T R^{\mathrm{S} / \mathrm{S}} ; \mathrm{Rrm}^{+/ T G}$ mice at $10 \mathrm{wk}$ of age analyzed by computerized tomography (CT). (F) Kaplan-Meyer curves of $A T R^{\mathrm{S} / \mathrm{S}}{ }_{;} \operatorname{Rrm}^{+/+}(n=25)$ and $A T R^{\mathrm{S} / \mathrm{S}}$; Rrm $2^{+/ T G}(n=26)$ mice. The $P$-value was calculated with the Mantel-Cox log rank test.

the transgene also increased the maximum life span of ATR hypomorphic mice from 54 wk to $91 \mathrm{wk}$. Collectively, these results demonstrate that increased RNR activity limits the consequences derived from reduced ATR activity in mammals.

Here we show that RNR activity can be enhanced in mammals by overexpression of the RRM2 subunit. A previous report that generated cDNA-based RNR transgenic mice revealed that broad overexpression of RRM2 increases the incidence of lung neoplasms (Xu et al. 2008). So far, we failed to detect a higher incidence of tumors on Rrm $2^{\mathrm{TG}}$ mice. It is possible that the difference between these studies relies on the fact that our transgenic RRM 2 is expressed under its endogenous promoter so that we are not overexpressing highly increased levels in tissues where RNR expression is normally low (such as the lungs). To further clarify this issue, we forced the development of lung tumors on Rrm $2^{\mathrm{TG}}$ mice by crossing them with a genetic model of lung carcinogenesis induced by the K-Ras oncogene (Guerra et al. 2003), where we also failed to see significant differences (Supplemental Fig. S5). Hence, the increased RNR activity of Rrm $2^{\mathrm{TG}}$ mice does not promote cancer, which is consistent with the fact that dNTPs are kept within normal levels in RRM2-overexpressing cells. In contrast, our work reveals that increased RRM2 levels can diminish the severity of the pathologies that lead to the premature aging and death of ATR mutant mice. Work in yeast has recently shown that increased RNR activity reduces genomic instability in several DNA repair 
mutants besides mec1s (Poli et al. 2012). To what extent increased RNR activity can also alleviate the phenotypes of additional genomic instability-driven diseases in mammals demands further investigation. Interestingly, folates, the nucleotide precursors, are routinely used in medicine for the prevention or treatment of a wide range of diseases, including various age-related pathologies. Our work suggests that strategies directed to increase RNR activity might also have beneficial effects in mammalian disease. Collectively, our data validate $\mathrm{Rrm} 2^{\mathrm{TG}}$ mice as a valuable mammalian model of increased RNR activity and demonstrate that ATR-dependent regulation of nucleotide pools contributes to the phenotypes of Seckel mice.

\section{Materials and methods}

\section{Mouse models}

For the generation of $\mathrm{Rrm} 2^{\mathrm{TG}}$ mice, a $26-\mathrm{kb}$ region from the mouse genome encompassing the Rrm2 gene was cloned from a BAC (RP23-460K8) into a minimal vector by recombineering (Gene Bridges) and was subsequently used for the generation of transgenic mice by microinjection into fertilized oocytes. ATR-Seckel (Murga et al. 2009) mice have been described.

\section{Nucleoside supplementation}

Where indicated, a nucleoside solution commercialized as a supplement for improving the growth of embryonic stem cells was used at the suggested dose (EmbryoMax ES Cell Qualified Nucleosides, Millipore).

\section{Cell vability assays}

The assay for measuring cell viability by HTM using TO-PRO-3 and Hoechst 33342 has been described before (Eguren et al. 2014).

Extended methods are in the Supplemental Material.

\section{Acknowledgments}

A.J.L.-C. and C.A. were funded a post-doctoral fellowship from the Spanish Association for Cancer Research (AECC). J.S. is a recipient of a predoctoral fellowship from the Spanish government (BES-2012-05 2030). S.V. and H. G. are funded by the Swedish Research Council and the Swedish Cancer Society. Work in O.F.-C.'s laboratory was supported by Fundación Botín, Banco Santander through its Santander Universities Global Division, and grants from Ministerio de Economía y Competitividad (MINECO; SAF2011-23753), Worldwide Cancer Research (12-0229), Fundació La Marato de TV3, Howard Hughes Medical Institute, and the European Research Council (ERC-617840). Work in A.J.L.-C.'s laboratory is funded by the Danish Council for Independent Research (DFF) and the Danish National Research Foundation.

\section{References}

Akerblom L, Ehrenberg A, Graslund A, Lankinen H, Reichard P, Thelander L. 1981. Overproduction of the free radical of ribonucleotide reductase in hydroxyurea-resistant mouse fibroblast $3 \mathrm{~T} 6$ cells. Proc Natl Acad Sci 78: 2159-2163.

Arnaoutov A, Dasso M. 2014. Enzyme regulation. IRBIT is a novel regulator of ribonucleotide reductase in higher eukaryotes. Science 345: $1512-1515$.

Barlow JH, Faryabi RB, Callen E, Wong N, Malhowski A, Chen HT, Gutierrez-Cruz G, Sun HW, McKinnon P, Wright G, et al. 2013. Identification of early replicating fragile sites that contribute to genome instability. Cell 152: 620-632.

Bester AC, Roniger M, Oren YS, Im MM, Sarni D, Chaoat M, Bensimon A, Zamir G, Shewach DS, Kerem B. 2011. Nucleotide deficiency promotes genomic instability in early stages of cancer development. Cell 145: 435-446.
Brown EJ, Baltimore D. 2000. ATR disruption leads to chromosomal fragmentation and early embryonic lethality. Genes Dev 14: 397-402.

Casper AM, Nghiem P, Arlt MF, Glover TW. 2002. ATR regulates fragile site stability. Cell 111: 779-789.

Cimprich KA, Cortez D. 2008. ATR: an essential regulator of genome integrity. Nat Rev Mol Cell Biol 9: 616-627.

D'Angiolella V, Donato V, Forrester FM, Jeong YT, Pellacani C, Kudo Y, Saraf A, Florens L, Washburn MP, Pagano M. 2012. Cyclin F-mediated degradation of ribonucleotide reductase $\mathrm{M} 2$ controls genome integrity and DNA repair. Cell 149: 1023-1034.

Danilova N, Bibikova E, Covey TM, Nathanson D, Dimitrova E, Konto Y, Lindgren A, Glader B, Radu CG, Sakamoto KM, et al. 2014. The role of the DNA damage response in zebrafish and cellular models of Diamond Blackfan anemia. Dis Model Mech 7: 895-905.

de Klein A, Muijtjens M, van Os R, Verhoeven Y, Smit B, Carr AM, Lehmann AR, Hoeijmakers JH. 2000. Targeted disruption of the cell-cycle checkpoint gene ATR leads to early embryonic lethality in mice. Curr Biol 10: 479-482.

Desany BA, Alcasabas AA, Bachant JB, Elledge SJ. 1998. Recovery from DNA replicational stress is the essential function of the S-phase checkpoint pathway. Genes Dev 12: 2956-2970.

Eguren M, Alvarez-Fernandez M, Garcia F, Lopez-Contreras AJ, Fujimitsu $\mathrm{K}$, Yaguchi H, Luque-Garcia JL, Fernandez-Capetillo O, Munoz J, Yamano H, et al. 2014. A synthetic lethal interaction between APC/ $\mathrm{C}$ and topoisomerase poisons uncovered by proteomic screens. Cell Rep 6: 670-683.

Eriksson S, Graslund A, Skog S, Thelander L, Tribukait B. 1984. Cell cycledependent regulation of mammalian ribonucleotide reductase. The $S$ phase-correlated increase in subunit M2 is regulated by de novo protein synthesis. J Biol Chem 259: 11695-11700.

Garcia-Cao I, Garcia-Cao M, Martín-Caballero J, Criado LM, Klatt P, Flores JM, Weill JC, Blasco MA, Serrano M. 2002. 'Super p53' mice exhibit enhanced DNA damage response, are tumor resistant and age normally. ЕМВО J 21: 6225-6235.

Guerra C, Mijimolle N, Dhawahir A, Dubus P, Barradas M, Serrano M, Campuzano V, Barbacid M. 2003. Tumor induction by an endogenous $\mathrm{K}$-ras oncogene is highly dependent on cellular context. Cancer Cell 4: $111-120$.

Huang M, Zhou Z, Elledge SJ. 1998. The DNA replication and damage checkpoint pathways induce transcription by inhibition of the Crt 1 repressor. Cell 94: 595-605.

Jordan A, Reichard P. 1998. Ribonucleotide reductases. Annu Rev Biochem 67: 71-98.

Lecona E, Fernandez-Capetillo O. 2014. Replication stress and cancer: it takes two to tango. Exp Cell Res 329: 26-34.

Lee YD, Wang J, Stubbe J, Elledge SJ. 2008. Dif1 is a DNA-damage-regulated facilitator of nuclear import for ribonucleotide reductase. Mol Cell 32: $70-80$.

Lewis WH, Wright JA. 1974. Altered ribonucleotide reductase activity in mammalian tissue culture cells resistant to hydroxyurea. Biochem Biophys Res Commun 60: 926-933.

Liu Q, Guntuku S, Cui XS, Matsuoka S, Cortez D, Tamai K, Luo G, Carattini-Rivera S, DeMayo F, Bradley A, et al. 2000. Chk1 is an essential kinase that is regulated by Atr and required for the $G_{2} / M$ DNA damage checkpoint. Genes Dev 14: 1448-1459.

Liu C, Powell KA, Mundt K, Wu L, Carr AM, Caspari T. 2003. Cop9/signalosome subunits and Pcu4 regulate ribonucleotide reductase by both checkpoint-dependent and -independent mechanisms. Genes Dev 17: $1130-1140$.

Lopez-Contreras AJ, Fernandez-Capetillo O. 2010. The ATR barrier to replication-born DNA damage. DNA Repair (Amst) 9: 1249-1255.

Lopez-Contreras AJ, Gutierrez-Martinez P, Specks J, Rodrigo-Perez S, Fernandez-Capetillo O. 2012. An extra allele of Chk1 limits oncogene-induced replicative stress and promotes transformation. J Exp Med 209: 455-461.

Lopez-Contreras AJ, Ruppen I, Nieto-Soler M, Murga M, RodriguezAcebes S, Remeseiro S, Rodrigo-Perez S, Rojas AM, Mendez J, Munoz J, et al. 2013. A proteomic characterization of factors enriched at nascent DNA molecules. Cell Rep 3: 1105-1116.

Monasor A, Murga M, Lopez-Contreras AJ, Navas C, Gomez G, Pisano DG, Fernandez-Capetillo O. 2013. INK4a/ARF limits the expansion of cells suffering from replication stress. Cell Cycle 12: 1948-1954.

Murga M, Bunting S, Montana MF, Soria R, Mulero F, Canamero M, Lee Y, McKinnon PJ, Nussenzweig A, Fernandez-Capetillo O. 2009. A mouse 
model of ATR-Seckel shows embryonic replicative stress and accelerated aging. Nat Genet 41: 891-898.

Poli J, Tsaponina O, Crabbé L, Keszthelyi A, Pantesco V, Chabes A, Lengronne A, Pasero P. 2012. dNTP pools determine fork progression and origin usage under replication stress. EMBO J 31: 883-894.

Seckel H. 1960. Bird-headed dwarfs: studies in developmental anthropology including human proportions. Charles C Thomas, Springfield, IL.

Sirbu BM, McDonald WH, Dungrawala H, Badu-Nkansah A, Kavanaugh GM, Chen Y, Tabb DL, Cortez D. 2013. Identification of proteins at active, stalled, and collapsed replication forks using isolation of proteins on nascent DNA (iPOND) coupled with mass spectrometry. I Biol Chem 288: 31458-31467.

Toledo LI, Murga M, Zur R, Soria R, Rodriguez A, Martinez S, Oyarzabal J, Pastor J, Bischoff JR, Fernandez-Capetillo O. 2011. A cell-based screen identifies ATR inhibitors with synthetic lethal properties for cancer associated mutations. Nat Struct Mol Biol 18: 721-727.

Tonin PN, Stallings RL, Carman MD, Bertino JR, Wright JA, Srinivasan PR, Lewis WH. 1987. Chromosomal assignment of amplified genes in hydroxyurea-resistant hamster cells. Cytogenet Cell Genet 45: 102-108.

Vallen EA, Cross FR. 1999. Interaction between the MEC1-dependent DNA synthesis checkpoint and G1 cyclin function in Saccharomyces cerevisiae. Genetics 151: 459-471.
Wu X, Huang M. 2008. Dif1 controls subcellular localization of ribonucleotide reductase by mediating nuclear import of the R2 subunit. Mol Cell Biol 28: 7156-7167.

Xu X, Page JL, Surtees JA, Liu H, Lagedrost S, Lu Y, Bronson R, Alani E, Nikitin AY, Weiss RS. 2008. Broad overexpression of ribonucleotide reductase genes in mice specifically induces lung neoplasms. Cancer Res 68: $2652-2660$.

Zeman MK, Cimprich KA. 2014. Causes and consequences of replication stress. Nat Cell Biol 16: 2-9.

Zhang YW, Jones TL, Martin SE, Caplen NJ, Pommier Y. 2009. Implication of checkpoint kinase-dependent up-regulation of ribonucleotide reductase R2 in DNA damage response. J Biol Chem 284: 18085-18095.

Zhao X, Rothstein R. 2002. The Dunl checkpoint kinase phosphorylates and regulates the ribonucleotide reductase inhibitor Smll. Proc Natl Acad Sci 99: 3746-3751.

Zhao X, Muller EG, Rothstein R. 1998. A suppressor of two essential checkpoint genes identifies a novel protein that negatively affects dNTP pools. Mol Cell 2: 329-340.

Zhao X, Chabes A, Domkin V, Thelander L, Rothstein R. 2001. The ribonucleotide reductase inhibitor Smll is a new target of the Mec1/Rad53 kinase cascade during growth and in response to DNA damage. EMBO J 20: 3544-3553. 


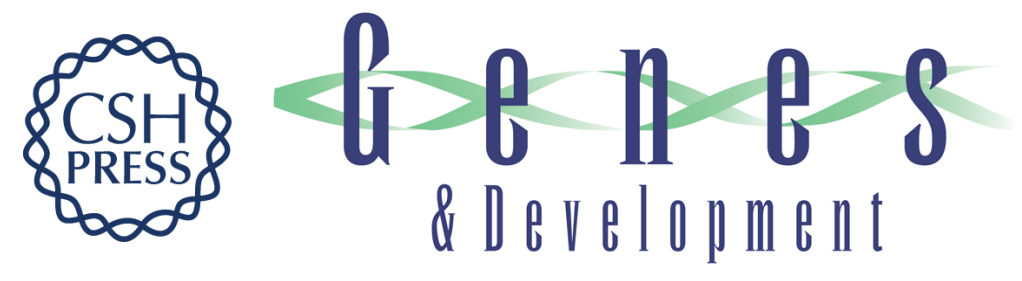

\section{Increased $\mathrm{Rrm} 2$ gene dosage reduces fragile site breakage and prolongs survival of ATR mutant mice}

Andres J. Lopez-Contreras, Julia Specks, Jacqueline H. Barlow, et al.

Genes Dev. 2015, 29:

Access the most recent version at doi:10.1101/gad.256958.114

\section{Supplemental http://genesdev.cshlp.org/content/suppl/2015/04/01/gad.256958.114.DC1 Material}

References

Creative

Commons

License

Email Alerting

Service
This article cites 39 articles, 18 of which can be accessed free at: http://genesdev.cshlp.org/content/29/7/690.full.html\#ref-list-1

This article is distributed exclusively by Cold Spring Harbor Laboratory Press for the first six months after the full-issue publication date (see

http://genesdev.cshlp.org/site/misc/terms.xhtml). After six months, it is available under a Creative Commons License (Attribution-NonCommercial 4.0 International), as described at http://creativecommons.org/licenses/by-nc/4.0/.

Receive free email alerts when new articles cite this article - sign up in the box at the top right corner of the article or click here.

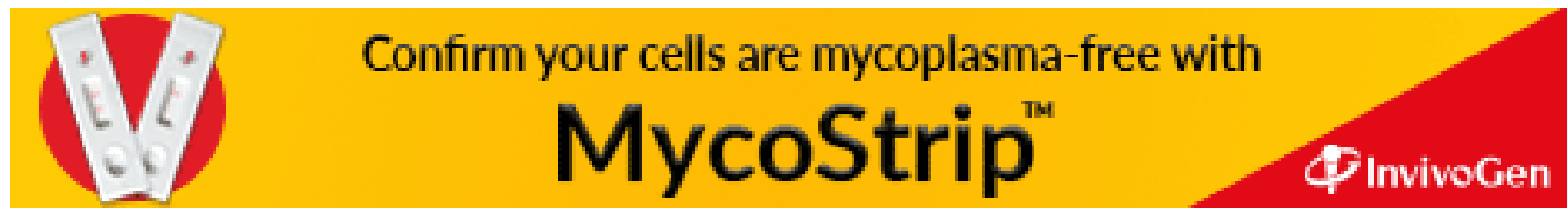

